Special issue of the 2nd International Conference on Computational and Experimental Science and Engineering (ICCESEN 2015)

\title{
A Study of Evaluation of Solar Energy Simulation and Modeling Systems
}

\author{
Z. $\mathrm{ER}^{*}$ \\ Istanbul Technical University, Faculty of Science and Letters, Physics Engineering Department (13b), \\ 34469 Maslak, Istanbul, Turkey
}

\begin{abstract}
In the solar energy application, there are several obstacles during the design of projects. The investors are still enforcing with deliberately carrying out the right and feasible project decision. In this point simulation techniques have been emerging their indispensable importance. Thereby the simulation can be used as an adaptive purpose not only during the design process of a plant but also for the existing plants efficacy while determining operational condition of solar system on a daily basis by probabilistic methods due to random characteristics of the meteorological data. This study mainly consists two parts. The first part of this study overarches the review of existing software tools and models which are reachable at the preparation time of this study. Then the reviewed software tools and models were classified briefly in the figures. The second part of the study outlines the developed new code in $\mathrm{C} \#$ computer programming language for the solar calculations. The results which were obtained framed the developed code, were evaluated with the convenient existing software tools and models for assessment of their correlation and also the results were demonstrated with graphs for their utilization. Consequently the software systems were enlightened that are mostly used in scientific projects including solar software simulation packages and modeling background together with comparison of separate or combined PV software programs by their field of application which would bolster further research studies.
\end{abstract}

DOI: 10.12693/APhysPolA.130.72

PACS/topics: 07.05.Tp, 07.05.Bx, 07.05.Kf

\section{Introduction}

The study of complex systems in various areas of science and engineering has revealed the necessity to complement the conventional experiments with theoretical methods based on computer simulation. Computer simulation is a powerful tool extensively used for investigating efficiency of solar systems from performance analysis models implementation. The performance analysis models of solar systems are characterized through their structure and intensity interactions. Therefore computer simulations bridge pre-recognition on the improvement of efficiency in real life behavior.

Solar simulations generate information such as sun positions and solar angles for solar system performance necessary to quantify the solar radiation and transport properties of a solar energy system [1-4]. Calculation properties include the angles, estimation of solar radiation, and thermal analysis. The direct calculations of thermal analysis and estimation of solar radiation are out of the aim of this paper. The simulation method can be performed in three main steps. Location and date are the input parameters of the simulation. Thus, the system evolves through solar calculation equations, enabling sun angles to estimation of solar radiation in the simulation tools. Solar radiation as functions of locations and date are calculated for their interpretation in terms of solar angles and surface orientation. The proper loca-

*e-mail: erzuh@itu.edu.tr tion choice is the first and the very essential step in solar system design procedure. If the location is not properly chosen, the most carefully planned solar system would not probably work in its short-run and long-run. Before installing any solar system, the direction, angle and location of the solar collector must be determined. The most fundamental requirement is exposing the solar collector to the most sunlight possible each day and throughout the year $[3,5-7]$.

The purpose of algorithms is to acquaint the software user with the basic terminology, concepts, and formulae needed to understand and utilize solar data. This is accomplished in this study of explaining parameters needed to calculate the position of the sun in the sky. To assess the availability of solar radiation at different locations, measurements of global radiation, diffuse radiation, beam radiation, sun shine hours, bright sun shine hours, maximum and minimum temperature, humidity, pressure, visibility, wind speed and direction, gust speed, water precipitation, and air mass are very important parameters [8-10]. In this study the calculations has been computed by the algorithm that has been developed in $\mathrm{C} \#$ to determine solar angles due to the given location and date.

\section{General forms of solar calculations}

The exact time of sunrise and sunset will be useful not only for people but also for the energy system operations at the junction of day and night. It is important for engineers to have working knowledge of the earth relationship to the sun. The fundamental unit of solar time is day. The solar time can be classified in two types which are 
called apparent true solar time (sundial time) and mean solar time (clock time). Sunrise is the time when the upper part of the Sun is visible, and sunset is when the last part of the Sun is about to disappear below the horizon (in clear weather conditions) $[1,2,6,7]$.

Radiation data depends on the solar basics with respect to the meteorological and astronomical evaluations such as calculations of solar angles. On the other hand, accurate quantitative data of the variation of solar radiation reaching the earth surface, together with relevant meteorological parameters are essential requirements for a wide range of scientific studies.

The solar radiation on the Earth surface is defined with respect to the Earth rotation around own axis and Sun orbit which leads the angles between radiations from the Sun and the Earth surface. Sun position can be described by the angles. As to benefit effectively of radiations, there are two of fundamental terms of solar energy necessary for accurate prediction of the Sun position described by the angles: the solar altitude and the solar azimuth. Above these two angles the declination angle and hour angle need to be defined $[1,2,8]$. The azimuth displayed is the horizontal direction of the Sun at sunrise and sunset, since the times used for the sunrise and sunset calculations are also used for azimuth calculation.

In the literature, several methods are existing for modeling solar radiation components (global, beam, and diffuse) on the ground of parametric models and decomposition models. The trigonometric correlation between zenith, declination, azimuth and hour angles is as below. The solar altitude angle $(\alpha)$, solar declination angle $(\delta)$, sunrise hour angle $\left(\omega_{\mathrm{s}}\right)$, solar azimuth angle $\left(\gamma_{\mathrm{s}}\right)$ and sunset and sunrise $\left(\omega_{0}\right)$ were computed by Equation 1, Equation 2, and Equation 3 respectively [6, 7, 9, 10]. These equations are set on the new developed code in this study.

$$
\begin{aligned}
& \sin \alpha=\sin \phi \sin \delta+\cos \phi \cos \delta \cos w, \\
& \delta=23.45^{\circ} \sin \left(\frac{360(284+n)}{365}\right), \\
& w_{\mathrm{s}}=\cos ^{-1}(-\tan \phi \tan \delta), \\
& \tan \gamma_{\mathrm{s}}=\frac{\sin \omega}{\sin \varphi \cos \omega-\cos \varphi \tan \delta}, \\
& \omega_{0}=\cos ^{-1}(-\tan \varphi \tan \delta),
\end{aligned}
$$

where $n$ is the number of days of the year starting from first January, $\varphi$ is the latitude of the related location (in degrees).

\section{Solar simulation}

There is a need to obtain solar radiation data which is serviced from a current meteorological information system for several solar studies such as building of solar power plant [10]. First of all, in this study there have been reviewed the meteorological data information systems to get open sources data which are available in different parameters. Research and reviews as a result of several web pages have been carried out to determine the site called and another software tools are required that is software components to communicate with each other. It was observed that the other application is needed to write an existing application that will interface. On the other hand, a vehicle that will be created needs to be able to use software defined methods on a web site which supplies open source meteorological data. In the light of these observations, this study was aimed particularly to the comparison of software tools of solar calculation then after this study was concentrated on preparation of a new $\mathrm{C} \#$ code that will do the solar calculations to make projection for solar forecasting.

The developed code in this study has also been compared with the professional tools and the commonly used software to be able to determine its robustness. In these premises, the brief introduction of the commonly used tools has been classified and summarized in the following paragraphs to depict their field of expertise and their field of implementation.

Naturally, software tools are covering different categories for their specific field of application like simulation, analysis, planning, economic evaluation, monitoring and so forth $[3,11-13]$. In this study as it was underlined here, investigated software tools are classified under three major categories that would be coined as: "Simulation", "Economic Evaluation" and the "Data Analysis and Planning". First and the foremost, this paper explicitly hereby underlines that this study is never aiming to neither the advertisement nor the marketing of any software tool(s) that are commonly used in solar industry that were demonstrated in the following tables. Thereto, the framework of this study was not designed to provide any consultancy either to the makers or their potential users. The sole aim of this study is to present the alternative software tools with their classification to those ones who are concerned or interested in solar energy applications. The following figures and table are demonstrating the taxonomy of solar software tools depending upon their classification based on their primer purpose. Figure 1 demonstrates the software tools which are most commonly used for simulation together with backward and forward judgement on design parameters while their brief descriptions underline their abilities.
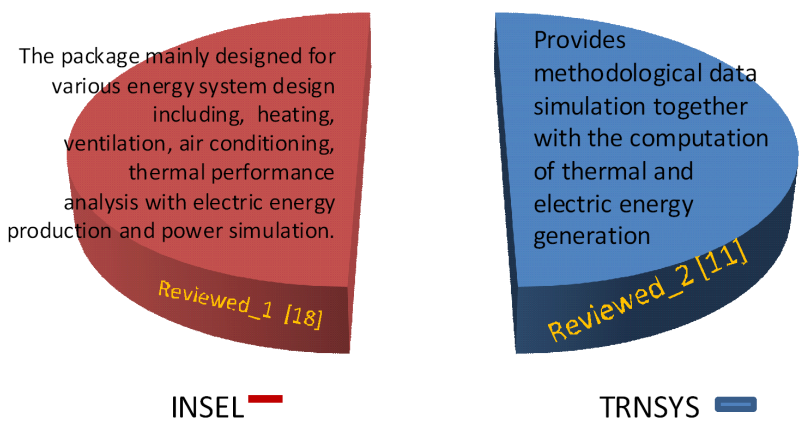

Fig. 1. Multi purpose simulation category for solar application.

Figure 2 displays the economic evaluation tools for 


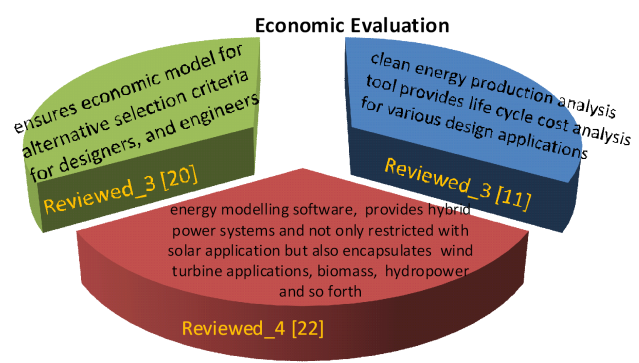

- RET screen - HOMER

= Solar Advisor Model

Fig. 2. Economic evaluation category for solar application.

solar power plants while providing the opportunity to benchmark efficiency with alternative energy production abilities and their probable coupled output applications.

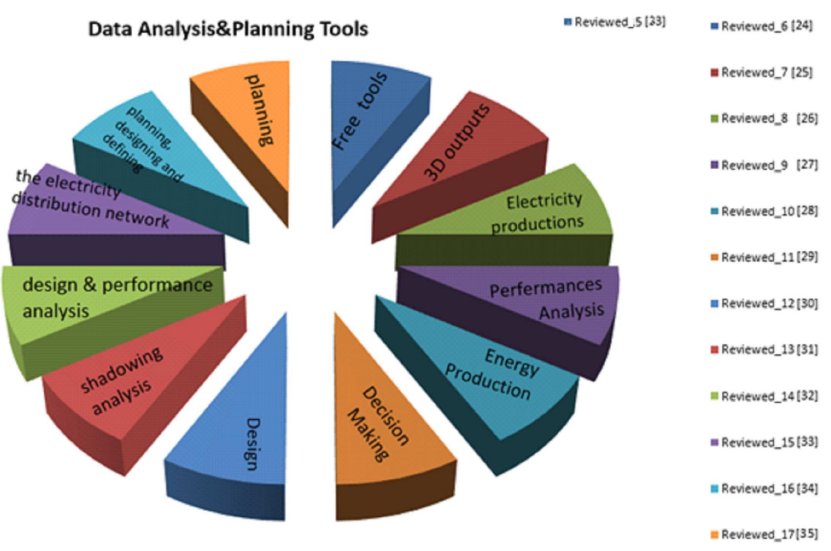

Fig. 3. Data analysis and planning category for solar application.

Figure 3 mainly concentrates on the software tools designed for data analysis for the selection of appropriate PV technologies. Therefore several of them are also providing financial assessment model together with their simulation opportunities.

Therefore the above stated issues have underlined improvement of solar plants by taking into account both the variation of the incidence radiation direction during the day time and the variation of the declination angle among the year, providing facilities to benefit from the solar energy [14-16]. Therefore, solar calculations which consist of important parameter that is called solar radiation depending on time and angles, is the first step for simulation studies $[1,7,17,18]$.

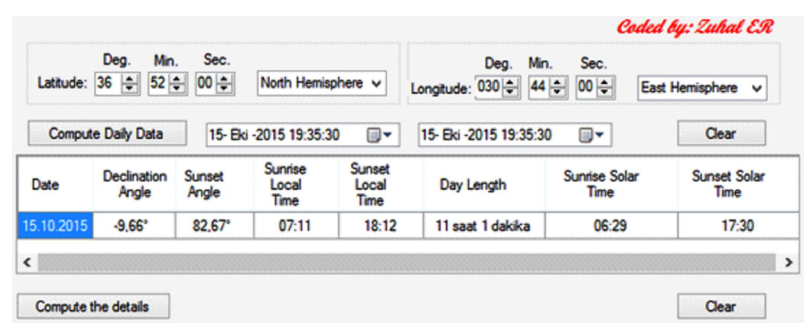

Fig. 4. Screen view of the simulation code outputs.

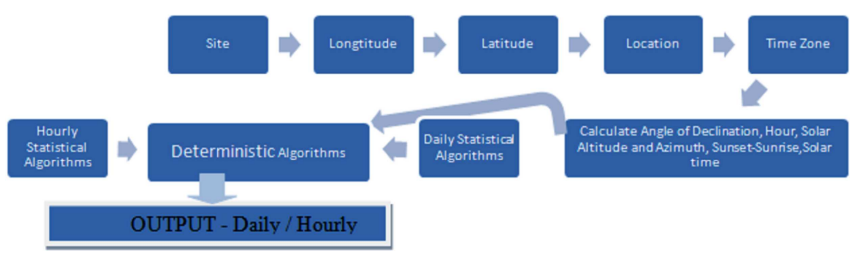

Fig. 5. Simulation methodology.

From this point of view this study was aimed to prepare a new software code for the solar calculations. The developed code has layers within classes and methods. Therefore, the object oriented base solar calculations have been developed using $\mathrm{C} \#$ Code. Examples of the methods in this study are underlined below. Screen view of the simulation code outputs and the simulation methodology of the system was illustrated in Fig. 4 and Fig. 5, respectively. The core of the developed code consists of two classes whose their method signatures are illustrated below.

\section{- public static void Values_DailyCalculations}

Method of Values DailyCalculations has parameter values of calendar, altitude, latitude, declination angle, sun set degree, sun clock sun rise hour, sun clock sun set hour, standard clock sun rise hour, standard clock sun set hour, day length and time difference, respectively.

- public static void VariableValues DailyCalculations

Method of VariableValues DailyCalculations has parameter values of time difference, declination angle, altitude, clock angle, sun elevation angle and azimuth angle, respectively.

The user should select the latitude and longitude from dropdown user interface row in the developed code. When clicking the button of Compute Daily Data, request is done for calculations. Then, this $\mathrm{C} \#$ Code is going to format object and write it text area as shown in Fig. 4. User can see the date, declination angle, sunset angle, sunrise local time, sun set local time, day length, sunrise solar time and sunset solar time of defined date with their respective time intervals.

\section{Results and discussion}

The classification of solar software tools have been divided into three main categories in this study. It is evident that the taxonomy may be enriched; however their multi-application utilities could be the subject of more than one category which could easily cause confusion for selecting the appropriate type of software tools. That was why the above classification was restricted to three categories. It is evident that application categories would additionally encapsulate smart phone applications, monitoring and control tools, site management tools, site analysis tools, solar radiation maps and data, online based 
tools, website based applications and so forth. Nevertheless, their main sole service is concentrated on providing service either for simulation or for economic evaluation or selection of appropriate design and data analysis for decision making and planning or their various combinations depending on the design of software packages provided by the makers. Therefore it is believed that the simple classification would ensure more concrete identification for the users rather than their application platform(s) classification.

All in all, the listed suppliers and their products' brief description that were demonstrated in Figs. 1-3, respectively, are not obviously demonstrating all gamut of professionally prepared software tools. They have been selected for sampling the solar power shelf products and their ability for their utilization during design, modeling, simulation, economic analysis etc. It is believed that there are umpteen products available both in local and international market to provide tailor made solutions to the designers, engineers and decision makers, prior to realization of solar power plant projects.

In the light of all above information, solar power derived from the system varies depending on the supply energy from the Sun, trigonometrically terms between the Sun and surface of system and meteorological data. Therefore, knowledge of the solar radiation data is an important parameter in the design of systems operating on solar energy for specified region. These parameters can be defined with preparation of suitable correlation for some region where the solar radiation data was not available.

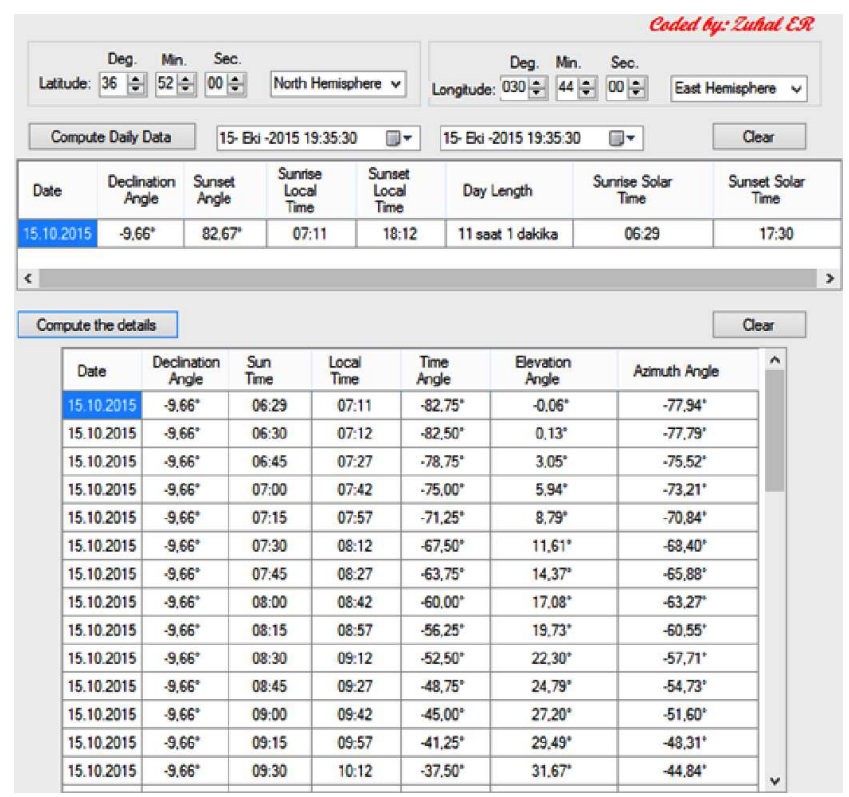

Fig. 6. The sample outputs of the code for Antalya.

This study gives answer to the question of what factors in today research forecasting need to be improved to get accurate irradiance. The originality of this study yields with the comparison of developed code performance with the existing software tools and models. The developed code enables the users to visualize the solar performance data by clicking simplest designed buttons where its mobile application could be also in service. When user click the Compute The Detail button, this $\mathrm{C} \#$ Code servers data such as sun time, local time, elevation angle and azimuth angle as shown in Fig. 6. Whether meteorological data such as humidity, wind etc. cannot be used this C\# Code will not show current day calculations. This C\# Code also supplies another simplicity with calculations which can be written in tables in Excel program. Therefore, radiation ratio according to date can be seen in graph as illustrated in Figs. 7-9.

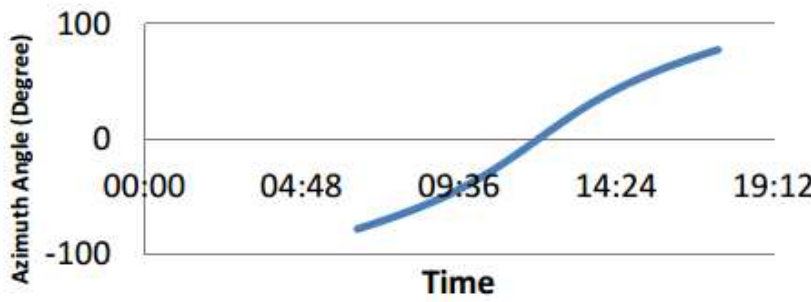

Fig. 7. Calculation of azimuth angle via $\mathrm{C} \#$ Code on 15 October 2015 in Antalya.

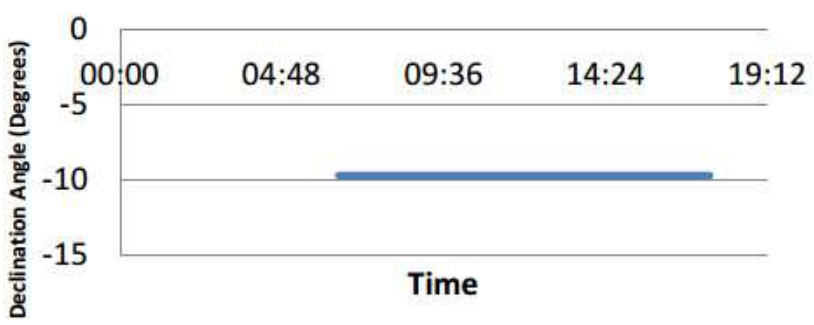

Fig. 8. Calculation of declination angle via $\mathrm{C} \#$ Code on 15 October 2015 in Antalya.

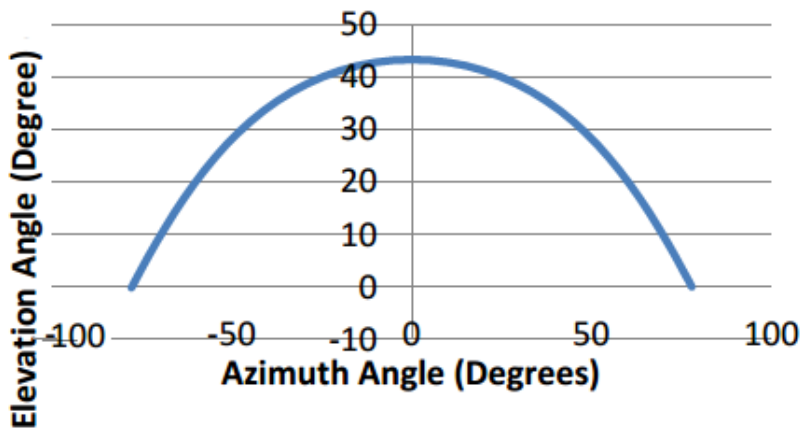

Fig. 9. Calculation of sun elevation angle via $\mathrm{C} \#$ Code on 15 October 2015 in Antalya.

The output of this study via simulation method that was coded by the author has been demonstrated in Figs. 7-9 for Antalya as a sample, whose outputs have been estimated and underlined on October 15, 2015 for the prediction of near future variables interactions. Lati- 
tude and longitude for Antalya are $36^{\circ} 52^{\prime} \mathrm{N}$ and $30^{\circ} 44^{\prime} \mathrm{E}$, respectively [17].

The sample outputs of the code for Antalya to give a sense of how the simulation is working are shown in Fig. 6.

Solar calculation studies which are background of forecasting would allow system operators more certainty about what the weather means for today peak solar output or tomorrow overall solar production. A case study was considered as well for demonstration purpose in this study to calculate the solar parameters for Antalya in Turkey. The modest simulation that was achieved in the study was coded with the same developed code while the benchmarking of the outputs were demonstrated in Fig. 10, where the results have been promising in $\pm 1 \%$ tolerance limits.

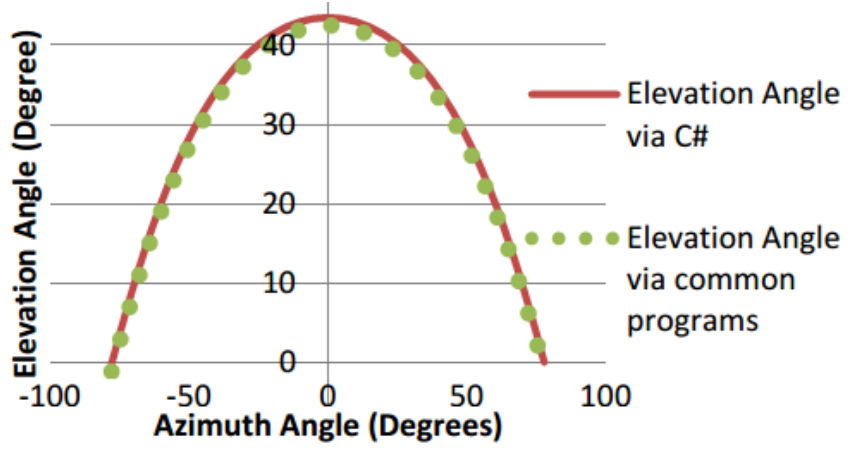

Fig. 10. Benchmarking of daily variation of solar elevation and azimuth for Antalya.

The elevation angle was obtained using the developed code and compared with the existing software tools. As shown in Fig. 10, it can be seen that the agreement between those data are good and this shows confidence in using the developed code for such calculations.

\section{Conclusions}

In this study, the new developed code and reviewed existing tools determine the daily or monthly solar calculation data depending on various parameters such as different weather conditions, sunshine hours, latitude of the given region. But there is always a trade-off between complexity and computational efficiency because the physics are so complicated you cannot model these things to a very high resolution.

This study represents the significance of the developed new code and the existing software tools that were designed to be used for solar power plant systems especially for the users who can readily adapt his or her own raw data to produce rough output for a project. Then the detailed parameters would be applied to those tools in scrutiny iteratively to get the most preferable design output contingent on the drawbacks that might be reckoned with particularly due to the application location of the solar plant together with the available infrastructure options of the actual site. Therefore the need for accurate climate data would be obtained by the data providers such as Google or local meteorological stations. The collection of intensive amount of data including the each seasonal conditions and their input to software would be a considerable amount of adversity unless the software tool has the interface to precisely adapt those data. Some software packages have the ability to provide shade reports and design reports taking into consideration of the appropriateness of input data while reporting the worst case, normal case and better case scenarios and their alternate combination for decision makers to assist them to select the most appropriate project. This does not actually be apprehended similar to the philosophy of a SWOT (strengths, weaknesses, occasions and threats) analysis; however most of the software tools provide quick judgement criteria while several of them are carrying out this event together with its financial assessment. Thereto most of the solar software tools are allowed to provide calculate the yield of a solar system over the annual cycle.

The new developed code in this study overarches simplest calculation with tolerance limits of $\pm 1 \%$. The truth that makes the new developed code originality is for the most part this tolerance limits whose acceptable results show suitable approaches to compare. Software applications does not depend on the life time and most of time the same case study was tested with accepted accurate results.

Last but not least, this study would like to point out and underline hereby again, all products that their names and access url(s) (uniform resource locator) have been identified as sample for the readers, should they incept to evaluate which would be more convenient to their specific application. It was aimed to keep this study in equal distance to all those products listed or not listed in this study and neither of them is aimed to be advertised while it is believed their pros and cons sorting are not the sole concern of this study. The further readings on these issues have been underlined in the references with which one may concentrate more over for his or her own prerequisites. The utilization of solar power, particularly the $\mathrm{PVs}$, are still promising and are going to be more sustainable in future by technological enhancement of their production together with cost reduction for the sake of preserving this planet for the future generations.

\section{References}

[1] N.C. Bezir, I. Akkurt, N. Ozek, En. Source A 32, 995 (2010).

[2] N.C. Bezir, I. Akkurt, N. Ozek, En. Source A 32, $512(2010)$.

[3] RET Screen Software Online User Manual, www. retscreen.net Clean Energy Decision Support Centre, 2005.

[4] HOMER, Getting Started Guide for Homer Legacy, Version 2.68, January 2011. 
[5] E.M. Nfah, J.M. Ngundam, M. Vandenbergh, J. Schmid, "Simulation of off-grid generation options for remote villages in Cameroon", a power point document www.usaee.org/usaee2015/.../RCiez_USAEE_ 10_27_15.ppt.

[6] Z. Er, Acta Phys. Pol. A 128, B-300 (2015).

[7] Z. Er, Acta Phys. Pol. A 128, B-477 (2015).

[8] Z. Er, B. Turna, Acta Phys. Pol. A 129, 865 (2015).

[9] C.K. Pandey, A.K. Katiyar, J. En. 2013, 305207 (2013).

[10] I.H. Altas, A.M. Sharaf, in: Proc. ICCEP '0\%, Int. Conf. on Clean Electrical Power, 2007, p. 341.

[11] TRNSYS 17, ATRaNsientSYstem Simulation program, Solar Energy Laboratory, Univ. of WisconsinMadison,sel.me.wisc.edu/trnsys (Reviewed_2).

[12] PVGis, Online free solar photovoltaic energy calculator for stand-alone or connected to the grid PV systems and plants, in Europe, Africa and Asia. Solar electricity generator simulation and solar radiations maps.

[13] PVresources, Domain where information on photovoltaic technologies and applications.
[14] D.K. Sharma, V. Verma, A.P. Singh, Int. J. Curr. Eng. Technol. 4, 725 (2014).

[15] IEA PVPS, Trends 2014 in Photovoltaic Applications, Survey Report of Selected IEA Countries between 1992 and 2013, Report IEA-PVPS T1-25:2014, 2014.

[16] S. Scampoli, ANSYS Advantages, Excell. Eng. Simulat.-Advantage 9, 46 (2015).

[17] www.solar.udel.edu/ELEG620/02_Solar_ radiation09.pdf.

[18] www.iaea.org/inis/collection/ NCLCollectionStore/_Public/38/106/38106953. pdf.

[19] Antalya Weather Information, www.antalya. climatemps.com/map.php. 